\title{
$B$-SETS AND COLORING PROBLEMS
}

BY S. K. STEIN

Communicated by Victor Klee, March 6, 1970

The set-theoretic concept of " $B$-set" was first used by Bernstein in 1908 in dealing with a topological question. Since then it has appeared in number theory, combinatorics, and logic. We consider it in the realm of linear graphs, and in particular, questions of map coloring.

1. Definitions. Let $F=\left\{X_{\alpha}: \alpha \in A\right\}$ be a family of sets. A set $B$ is called a $B$-set for this family if $B \cap X_{\alpha} \neq \varnothing$, all $\alpha \in A$, and $B \nsupseteq X_{\alpha}$, all $\alpha \in A$. A family $F$ need not have a $B$-set. Observe that if $B$ is a $B$-set for $F$, then so is the complement of $B$.

Consider a map covering $S_{2}$, the two-dimensional sphere. We shall assume that it is regular, that is, each vertex is of degree three. Each region of the map is a topological cell. Two regions are adjacent if they share at least one edge. A sequence of distinct regions $R_{1}$, $R_{2}, \cdots, R_{n}, n \geqq 3$, is a cycle of length $n$ if $R_{i}$ is adjacent to $R_{i+1}$, $1 \leqq i \leqq n-1$, and $R_{n}$ is adjacent to $R_{1}$. The cycle is odd or even according as $n$ is odd or even.

2. Statement of results. It is well known that a map can be colored with four colors such that adjacent regions have different colors if and only if the regions can be partitioned into two sets, neither of which contains an odd-cycle. For purposes of comparison, we record this as

THEOREM 1. A map can be colored with four colors if and only if the family of odd cycles has a $B$-set.

This theorem suggests that we determine which families of cycles of regions in a map have a $B$-set. The next two theorems treat extreme cases.

THEOREM 2. The family of cycles around vertices (hence of length 3 ) has $a$ B-set if and only if the edges can be labelled $r$ and $b$ in such a way that at each vertex are one $r$ and two $b$ 's.

That such $r, b$ labellings exist is a classical result of Petersen.

AMS Subject Classifications. Primary 0540, 0555, 5510.

Key Words and Phrases. B (Bernstein)-set, coloring, four-coloring, Petersen's theorem, cycles, Hamiltonian circuit, Whitney's theorem. 
THEOREM 3. The family of all cycles has a B-set if and only if there is a Hamiltonian circuit through the vertices of the map.

This contradicts an assertion made in [2, p. 616]. (The counterexample presented there has three regions with the same label surrounding a vertex on the lower base.)

Theorem 3 implies that if a particular map lacks a Hamiltonian circuit through its vertices, then the (finite) family of cycles in the map lacks a $B$-set. Indeed, as one example, we can show that in the map described by Tutte [3] the family of 3-, 4-, and 5-cycles has no $B$-set.

If we consider only cycles that surround a single region, we may deduce, from Whitney's Theorem [4] on the existence of Hamiltonian circuits through the vertices of the dual of the map, the following result.

THEOREM 4. Let $M$ be an infinite map of compact regions covering the Euclidean plane. Assume that $M$ is regular and that the union of any two adjacent regions is simply connected, and that the only three-cycles are those around $a$ vertex. Then the family of cycles around single regions has a B-set.

While these results were suggested by the four-color conjecture, the next theorem is a strengthening of the five-color theorem, and is proved similarly. It concerns not $B$-sets but a slight generalization, partitions of the regions into three families.

THEOREM 5. It is possible to partition the family of regions of a regular map into three sets in such a way that one set consists of isolated regions, while neither of the other two contains a cycle.

This theorem is clearly stronger than the five-color theorem and the theorem in [1] that asserts that there is a partition into three sets, none of which contains a cycle.

\section{REFERENCES}

1. Gary Chartrand, Hudson V. Kronk and Curtiss E. Wall, The point-arboricity of a graph, Israel J. Math. 6 (1968), 169-175. MR 38 \#4347.

2. Gary Chartrand and Hudson V. Kronk, The point-arboricity of planar graphs, J. London Math. Soc. 44 (1969), 612-616. MR 39 \#1350.

3. W. T. Tutte, On Hamiltonian circuits, J. London Math. Soc. 21 (1946), 98-101. MR 8, 397.

4. H. Whitney, A theorem on graphs, Ann. of Math. (2) 32 (1931), 378-390.

University of California, Davis, California 95616 